
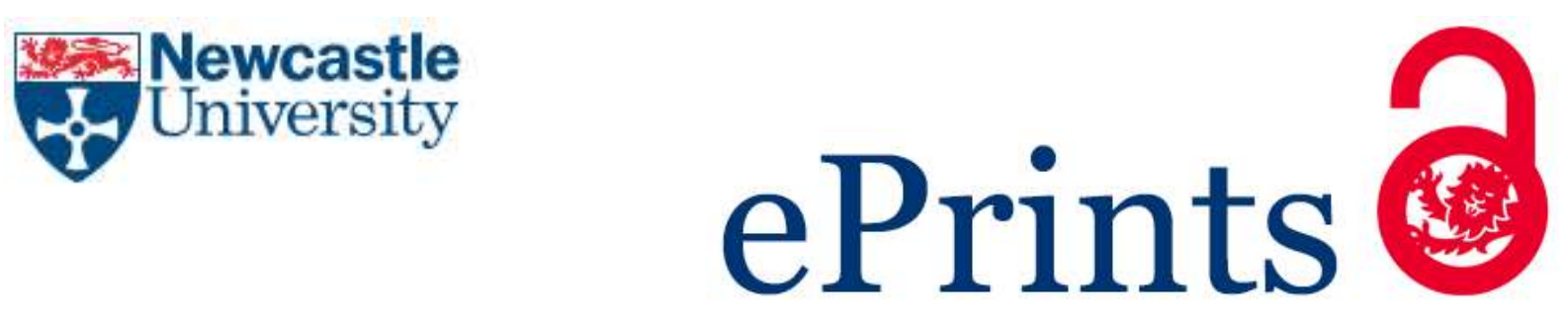

Turan O, Yigit S, Chakraborty N. Effects of wall heating on laminar mixed convection in a cylindrical enclosure with a rotating end wall. International Journal of Thermal Sciences 2018, 131, 80-93.

\title{
Copyright:
}

(C) 2018. This manuscript version is made available under the CC-BY-NC-ND 4.0 license

DOI link to article:

https://doi.org/10.1016/i.ijthermalsci.2018.05.005

Date deposited:

$29 / 05 / 2018$

Embargo release date:

26 May 2019

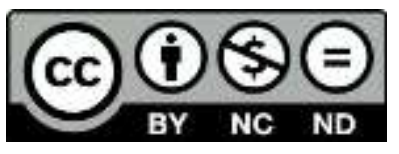

This work is licensed under a

Creative Commons Attribution-NonCommercial-NoDerivatives 4.0 International licence 


\section{Effects of wall heating on laminar mixed convection in a cylindrical enclosure with a rotating end wall}

Osman Turan ${ }^{1,2, \bowtie}$, Sahin Yigit $^{2}$, Nilanjan Chakraborty ${ }^{2}$

${ }^{1}$ Department of Mechanical Engineering, Bursa Technical University, Bursa, 16310, TURKEY

${ }^{2}$ School of Mechanical and Systems Engineering, Newcastle University, Newcastle-Upon-Tyne, NE1 7RU, UK

${ }^{\otimes}$ corresponding author.

osman.turan@btu.edu.tr 


\begin{abstract}
Steady-state laminar mixed convection in a cylindrical enclosure has been numerically analysed for different values of Reynolds, Richardson and Prandtl numbers given by $500 \leq R e \leq 3000$, $0 \leq R i \leq 1$ and $10 \leq P r \leq 500$ respectively. The aspect ratio (i.e. height: radius $=A R=H / R$ ) of the cylindrical container is considered to be unity (i.e. $A R=H / R=1$ ). The bottom and top covers of the cylindrical enclosure are kept at different temperatures $\left(T_{C}<T_{H}\right)$, while the cylindrical surface is taken to be adiabatic. The simulations for rotating top and bottom cover configurations yield the same numerical values of the mean Nusselt number $\overline{N u}$ when the thermal boundary conditions are kept unaltered. For this reason, only rotating top hot wall (i.e. C1 configuration) and rotating top cold wall (i.e. C2 configuration) have been considered for this analysis. The mean Nusselt number $\overline{N u}$ has been found to assume higher values in the $\mathrm{C} 2$ configuration than in the $\mathrm{C} 1$ configuration. Moreover, it has been found that the variation of the mean Nusselt number with Richardson number in the $\mathrm{C} 2$ configuration is qualitatively different from that in the $\mathrm{C} 1$ configuration. The simulation data has been used to propose a correlation for $\overline{N u}$ for the range of $R e, R i$ and $\operatorname{Pr}$ considered here for both $\mathrm{C} 1$ and $\mathrm{C} 2$ configurations. In addition to this, a regime diagram has been proposed for the $\mathrm{C} 2$ configuration in order to demarcate different flow regimes.
\end{abstract}

Keywords: Mixed convection; Rotating end wall; Reynolds number; Prandtl number; Richardson number. 
NOMENCLATURE

\begin{tabular}{lll}
$a$ & {$[-]$} & Bridging function \\
$A R$ & {$[-]$} & Aspect ratio, $(A R=H / R)$ \\
$b$ & {$[-]$} & Bridging function \\
$c_{p}$ & {$[\mathrm{~J} / \mathrm{kgK}]$} & Specific heat at constant pressure \\
$e_{a}$ & {$[-]$} & Relative error \\
$f_{1}$ & {$[-]$} & General Function \\
$g$ & {$\left[\mathrm{~m} / \mathrm{s}^{2}\right]$} & Gravitational acceleration \\
$G r$ & {$[-]$} & Grashof number \\
$h$ & {$\left[\mathrm{~W} / \mathrm{m}^{2} \mathrm{~K}\right]$} & Heat transfer coefficient \\
$H$ & {$[\mathrm{~m}]$} & Height of cylindrical enclosure \\
$k$ & {$[\mathrm{~W} / \mathrm{mK}]$} & Thermal conductivity \\
$k_{0}$ & {$[-]$} & Correlation parameters \\
$m_{0}$ & {$[-]$} & Correlation parameters \\
$N u$ & {$[-]$} & Nusselt number \\
$\overline{N u}$ & {$[-]$} & Mean Nusselt number \\
$P r$ & {$[-]$} & Prandtl number \\
$q$ & {$\left[\mathrm{~W} / \mathrm{m}^{2}\right]$} & Heat flux \\
$Q$ & {$[\mathrm{~W}]$} & Heat transfer rate \\
$R$ & {$[\mathrm{~m}]$} & Radius of cylindrical enclosure \\
$R a$ & {$[-]$} & Rayleigh number \\
$R e$ & {$[-]$} & Reynolds number \\
$R i$ & {$[-]$} & Richardson number \\
$T$ & {$[\mathrm{~K}]$} & Temperature \\
$U$ & $(\mathrm{~m} / \mathrm{s})$ & Characteristic velocity scales in radial direction \\
$V$ & $(\mathrm{~m} / \mathrm{s})$ & Characteristic velocity scales in tangential direction \\
$V_{\phi}$ & {$[-]$} & Non-dimensional swirl velocity, $\left(V_{\phi}=v H / \alpha\right)$ \\
& & \\
\hline & &
\end{tabular}

\section{The Greek symbols}

$\begin{array}{lll}\alpha & {\left[\mathrm{m}^{2} / \mathrm{s}\right]} & \text { Thermal diffusivity } \\ \beta & {[1 / \mathrm{K}]} & \text { Coefficient of thermal expansion } \\ \dot{\gamma} & {[1 / \mathrm{s}]} & \text { Shear rate } \\ \delta, \delta_{t h} & {[\mathrm{~m}]} & \text { Hydrodynamic and thermal boundary layer thickness } \\ \theta & {[-]} & \text { Non- dimensional temperature, }\left(\theta=\left(T-T_{\mathrm{C}}\right) /\left(T_{\mathrm{H}}-T_{\mathrm{C}}\right)\right) \\ \mu & {\left[\mathrm{Ns} / \mathrm{m}^{2}\right]} & \text { Plastic viscosity } \\ v & {\left[\mathrm{~m}^{2} / \mathrm{s}\right]} & \text { Kinematic viscosity } \\ \rho & {\left[\mathrm{kg} / \mathrm{m}^{3}\right]} & \text { Density } \\ \tau & {\left[\mathrm{N} / \mathrm{m}^{2}\right]} & \text { Shear stress } \\ \Omega & {[1 / \mathrm{s})} & \text { Angular velocity } \\ \psi & {\left[\mathrm{m}^{2} / \mathrm{s}\right]} & \text { Stream function } \\ \Psi & {[-]} & \text { Non-dimensional stream function, }(\Psi=\psi / \alpha)\end{array}$




\section{Subscripts}

$\begin{array}{ll}a d v & \text { Advective } \\ C & \text { Cold wall } \\ \text { conv } & \text { Convective } \\ d i f f & \text { Diffusive } \\ H & \text { Hot wall } \\ \text { max } & \text { Maximum value } \\ r & \text { Radial direction } \\ r e f & \text { Reference value } \\ w f & \text { Condition of the fluid in contact with the wall } \\ z & \text { Axial direction } \\ \phi & \text { Tangential direction }\end{array}$




\section{INTRODUCTION}

The flow induced by rotating one of the covers of a cylindrical container has a wide range of applications (e.g. chemical processing, bio-chemical synthesis, polymer processing, food preparation, pharmacology). Mixed convection plays a vital role not only in heat transfer rate applications but also influences the mixing rate for low Reynolds number applications. Therefore, it is necessary to investigate heat transfer characteristics and flow structure in this configuration so that the rates of heat transfer and mixing can be optimised. However, heat transfer and mixing rates in this configuration depend on many parameters such as container geometry and the rotational speed of the cover. Two special cases of rotating flow problems are the flows on top a rotating disk and inside an enclosure with a rotating end cover. Theodore von Karman pioneered the analysis of flows on top a rotating disk and such flows are commonly referred to as von-Karman flows, and an extensive review of such flows is provided in Ref. [1]. In addition to this, flow in cylindrical enclosures with a rotating cover has also been extensively analysed from various different viewpoints due to its wide range of roles in a range of different engineering applications. Vogel [2,3], Ronnenberg [4] and Bertela and Gori [5] analysed fluid flows in cylindrical enclosures with a rotating end wall, and the findings of these studies [2-5] have subsequently been extended by Escudier [6] based on an experimental analysis where the criterion for vortex breakdown has been proposed in terms of aspect ratio $H / R$ and Reynolds number $\Omega R^{2} / v$. Fujimura et al. [7] also experimentally investigated the flow generated by rotating end walls in a cylindrical container in which the top cover was rotated at a higher angular velocity than angular velocities at which the bottom and the side walls are rotated. The effects of the differential rotations of the container walls on the vortex breakdown have been found to be significant by Fujimura et al. [7]. Besides these experimental studies, several numerical investigations [8-14] analysed fluid flows in cylindrical enclosures with a rotating end wall. One of the fist numerical investigation was carried out by Lugt and Haussling [8], who focused on calculating the single recirculation bubble and verifying numerically the experimental data of Vogel [2] and Ronnenberg [4]. In addition to this, another pioneering numerical investigation was performed by Lopez [9] who was the first to calculate the full extent of the flows observed by Escudier [6]. Most investigations involving rotating end wall focused on the investigation of flows in cylindrical containers until Pereira and Sousa [13], who investigated 
vortex breakdown generated by a rotating end wall within a conical container. The flow produced in a conical container by a rotating end wall has also been numerically analysed by Escudier et al. [14] who reported that vortex breakdown is suppressed beyond a certain angle of inclination of the sidewall for both convergent (increasing radius towards the rotating end wall) and divergent (decreasing radius towards the rotating end wall) geometries.

The analysis of heat transfer characteristics in cylindrical enclosures with a rotating cover received relatively limited attention [10-12]. Lee and Hyun [11] analysed the effects of Prandtl number on heat transfer rate in this configuration and revealed that Prandtl number has an important influence on the heat transfer characteristics and advective transport has been found to strengthen with increasing Prandtl number. Iwatsu [12] investigated the effects of Reynolds and Richardson numbers at $\operatorname{Pr}=1$, in the range of $100 \leq R e \leq 3000$, and $0 \leq R i \leq 1$, on the flow pattern and heat transfer rate for swirling flows in cylindrical enclosures with an aspect ratio of unity (i.e. $A R=H / R=1$ ), and a heated rotating top wall based on numerical simulations. The analysis by Iwatsu [12] revealed that advective (diffusive) transport strengthens (weakens) and accordingly, the mean Nusselt number increases with decreasing Richardson number.

There are four possible different configurations for the flows induced by rotating one of the covers of a cylindrical container depending on the boundary conditions, which are schematically shown in Fig. 1. Existing analyses on flow induced by the rotation of one of the end covers in a cylindrical enclosure have been summarised in Table 1, where the boundary conditions and the governing non-dimensional parameters for which these studies were conducted have also been summarised along with the nature of the investigation (i.e. whether it is experimental or numerical). It can be seen from Table 1, the majority of existing analyses focused only on the flow structure, and there is no existing analysis in which the all possible configurations are investigated in terms of heat transfer characteristics. For this purpose, the present study focuses on a detailed analysis of the heat transfer characteristics in cylindrical enclosures with a rotating end wall for different boundary conditions which are schematically shown in Fig. 1. A parametric analysis has been conducted to analyse the effects of Richardson, Reynolds and Prandtl 
numbers on heat and momentum transport for a range of Reynolds, Richardson, and Prandtl numbers (definitions are provided in Section 2) given by $500 \leq R e \leq 3000,0 \leq R i \leq 1$ and $10 \leq \operatorname{Pr} \leq 500$ respectively for an aspect ratio (height: radius) of unity (i.e. $A R=H / R=1$ ). In this respect, the main objective of the present paper is to demonstrate the influences of Reynolds, Richardson and Prandtl numbers on mixed convection induced by a rotating end wall in a cylindrical enclosure with an aspect ratio of unity for different boundary conditions.

The rest of the article will be organised as follows. The necessary mathematical background and numerical implementation will be discussed in the next section, which will be followed by a detailed scaling analysis. Following these sections, results will be presented and subsequently discussed. The main findings are summarised and conclusions are drawn in the final section.

\section{MATHEMATICAL BACKGROUND}

\subsection{Governing equations and boundary conditions}

In this study, the flow is assumed to be laminar, incompressible, steady and axisymmetric (i.e. twodimensional). The conservation equations in the cylindrical coordinate system take the following form for steady-state incompressible axisymmetric swirling flows:

\section{Mass conservation equation}

$$
\frac{\partial u}{\partial r}+\frac{u}{r}+\frac{\partial w}{\partial z}=0
$$

\section{Momentum conservation equations}

$$
\begin{array}{ll}
r: \quad \rho\left(u \frac{\partial u}{\partial r}-\frac{v^{2}}{r}+w \frac{\partial u}{\partial z}\right)=-\frac{\partial p}{\partial r}+\mu\left[\frac{1}{r} \frac{\partial}{\partial r}\left(r \frac{\partial u}{\partial r}\right)-\frac{u}{r^{2}}+\frac{\partial^{2} u}{\partial z^{2}}\right] \\
\phi: \quad \rho\left(u \frac{\partial v}{\partial r}+\frac{u v}{r}+w \frac{\partial v}{\partial z}\right)=\mu\left[\frac{1}{r} \frac{\partial}{\partial r}\left(r \frac{\partial v}{\partial r}\right)-\frac{v}{r^{2}}+\frac{\partial^{2} v}{\partial z^{2}}\right] \\
z: \quad \rho\left(u \frac{\partial w}{\partial r}+w \frac{\partial w}{\partial z}\right)=-\frac{\partial p}{\partial z}+\rho g \beta\left(T-T_{r e f}\right)+\mu\left[\frac{1}{r} \frac{\partial}{\partial r}\left(r \frac{\partial w}{\partial r}\right)+\frac{\partial^{2} w}{\partial z^{2}}\right]
\end{array}
$$

\section{Energy conservation equation}




$$
\rho c_{p}\left(u \frac{\partial T}{\partial r}+w \frac{\partial T}{\partial z}\right)=k\left(\frac{1}{r} \frac{\partial T}{\partial r}+\frac{\partial^{2} T}{\partial r^{2}}+\frac{\partial^{2} T}{\partial z^{2}}\right)
$$

where $\mu$ is the dynamic viscosity, $T_{\text {ref }}$ is the reference temperature for evaluating the buoyancy term $\rho g \beta\left(T-T_{\text {ref }}\right)$ in the momentum conservation equation in the vertical direction, and here $T_{\text {ref }}$ is taken to be the cold cover temperature $T_{c}$. In addition, thermo-physical properties (thermal conductivity, specific heat, viscosity etc.) are considered to be constant and independent of temperature in this analysis for the sake of implicitly.

The numerical investigation is carried out for an axisymmetric domain for different boundary conditions, which are schematically shown in Fig. 1 . The aspect ratio $(A R=H / R)$ of the cylindrical container is considered to be unity (i.e. $A R=H / R=1$ ). The bottom and top covers of the cylindrical enclosures are kept at different temperatures $\left(T_{C}<T_{H}\right)$, while the cylindrical surface is considered to be adiabatic in nature. The temperature difference between the top and bottom covers are maintained small enough to ensure that Boussinesq approximation remains valid. No-slip boundary condition and impenetrable walls indicate that all the velocity components are identically zero on the surface of the container and at the non-rotating end wall. For the rotating end wall, radial and axial velocity components are identically zero due to impenetrability and no-slip conditions, and the tangential velocity component is given by $=\Omega r$.

\subsection{Non-dimensional numbers}

The ratio between inertial and viscous forces represents the strength of the forced convection component in this analysis. This ratio can be quantified by the Reynolds number $R e$ which is defined as:

$$
R e=\frac{\rho \Omega R^{2}}{\mu}
$$

The natural convection component of mixed convection can be characterised by the Grashof number, which represents the ratio of buoyancy to viscous forces, in the following manner:

$$
G r=\frac{\rho^{2} g \beta \Delta T H^{3}}{\mu^{2}}
$$


Another important non-dimensional number is Rayleigh number, $R a$, which demonstrates that the ratio of the strengths of thermal transports due to buoyancy to thermal diffusion, which is defined here in the following manner:

$$
R a=\frac{\rho^{2} c_{p} g \beta \Delta T H^{3}}{\mu k}=\operatorname{GrPr}
$$

where $\operatorname{Pr}$ is the Prandtl number, which is defined as:

$$
\operatorname{Pr}=\frac{\mu c_{p}}{k}
$$

Prandtl number shows the ratio of momentum diffusion to thermal diffusion. The Prandtl number also represents the ratio of the hydrodynamic boundary layer to thermal boundary layer thicknesses. In addition to this, Richardson number is a well-known non-dimensional parameter for mixed convection, which is used to evaluate the relative importance of the natural to forced convection:

$$
R i=\frac{G r}{R e^{2}}=\frac{g \beta \Delta T H^{3}}{\Omega^{2} R^{4}}
$$

The rate of convective heat transfer is generally characterised by the heat transfer coefficient $h$, which is expressed in a non-dimensional form in terms of the Nusselt number $N u$, as:

$$
N u=\frac{h R}{k}
$$

and the heat transfer coefficient $h$ is defined as:

$$
h=\left|-k \frac{\partial T}{\partial z}\right|_{w f} \times \frac{1}{T_{w a l l}-T_{\text {reff }}} \mid
$$

where subscript 'wf' refers to the condition of the fluid in contact with the wall, $T_{\text {wall }}$ is the wall temperature and $T_{r e f}$ is the appropriate reference temperature, which can be taken to be $T_{C}\left(T_{H}\right)$ for the hot (cold) wall respectively. For this configuration the mean heat transfer coefficient $\bar{h}$ and $\overline{N u}$ are given by: $\bar{h}=\int_{0}^{R} h 2 \pi r d r / \pi R^{2}$ and $\overline{N u}=\bar{h} R / k$, respectively.

\subsection{Numerical implementation, grid-independency, and bench-marking}

In the current study, the conservation equations of mass, momentum and energy have been solved in the framework of a finite-volume method using a computational fluid dynamics (CFD) software ANSYSFLUENT, which was previously successfully used for simulations of the flows induced by rotating one 
of the end covers of a cylindrical container [14 and 15]. A second-order central difference scheme is used for the discretisation of the diffusive terms and a second-order up-wind scheme is used for the convective terms. Coupling of pressure and velocity is achieved using the well-known SIMPLE (SemiImplicit Method for Pressure-Linked Equations) algorithm [16]. The convergence criteria have been taken to be $10^{-7}$ for all the relative (scaled) residuals.

Three different non-uniform meshes M1 $(50 \times 50)$, M2 $(100 \times 100)$ and M3 $(200 \times 200)$ have been investigated for each configuration, and the normalised minimum grid spacing $\Delta_{\text {min,cell }} / R$ and grid expansion ratio $r_{e}$ for these meshes have been provided in Table 2. The numerical uncertainties for the mean Nusselt number $\overline{N u}$ for $R e=1000$ and $R i=0.1$ at $P r=100$ are shown in Table 2. Table 2 indicates that the maximum relative error levels $\left(e_{a}\right)$ between M2 $(100 \times 100)$ and M3 $(200 \times 200)$ are under $1.0 \%$. Based on this analysis, the simulations have been conducted using mesh M2 $(100 \times 100)$ for both configurations, which is found to be sufficient for providing high accuracy and computational efficiency. In addition to a grid-independency study, the simulation results have also been compared to the simulation data by Iwatsu [12] for different Richardson and Reynolds number values at $P r=1.0$. As shown in Fig. 2, the present simulations results remain in excellent agreement with the corresponding benchmark data reported by Iwatsu [12]. Moreover, for code validation in Fig. 3 the streamlines obtained from numerical simulations for $R e=1854$ and $A R=H / R=2$ have been compared to the experimental flow visualisations reported by Escudier [6] which was used for numerical code validation in several previous numerical studies $[14,17,18]$. It can be seen from Fig. 3 that the simulation accurately predicts both the occurrence of the primary and secondary recirculation bubbles as well as their size and location along the centerline. Based on the evidences presented in Figs. 2 and 3, the numerical implementation can be considered sufficiently accurate and appropriate for the analysis undertaken in this paper.

\section{SCALING ANALYSIS}


A scaling analysis has been carried out in order to elucidate the possible influences of Reynolds, Richardson, Rayleigh and Prandtl numbers on the mean Nusselt number. The wall heat flux in the thermal boundary layer can be scaled as:

$$
q \sim k \frac{\Delta T}{\delta_{t h}} \sim h \Delta T
$$

Using Eq. (11), the Nusselt number can be scaled as:

$$
N u \sim \frac{h R}{k} \sim \frac{R}{\delta_{t h}}
$$

or

$$
N u \sim \frac{R}{\delta} f_{1}(R e, R i, R a, P r)
$$

where $f_{1}$ is a function of $R e, R i, R a$ and $P r$, which accounts for the ratio of hydrodynamic to thermal boundary layer thicknesses (i.e. $\delta / \delta_{t h} \sim f_{1}(R e, R i, R a, P r)$ ).

In order to determine the hydrodynamic boundary thickness $\delta$, the order of magnitudes of inertial and viscous forces in the radial direction can be equated:

$$
\rho \frac{V^{2}}{R} \sim \frac{\tau}{\delta}
$$

The shear stress can be scaled as $\tau \sim \mu(U / \delta)$ and thus Eq. (13) can be rewritten as:

$$
\rho \frac{V^{2}}{R} \sim\left(\mu \frac{U}{\delta}\right) \frac{1}{\delta}
$$

Using Eq. (14), hydrodynamic boundary thickness $\delta$ can be estimated as:

$$
\delta \sim \sqrt{\frac{\mu U R}{\rho V^{2}}}
$$

Here, $U$ and $V$ are the characteristic velocity scales in radial and tangential directions respectively, and they can be estimated as shown in Table 3 for different flow conditions. Eq. (15) can be rewritten by using the velocity scale $U \sim a(\Omega R)+b(\sqrt{g \beta \Delta T R})$ as follows:

$$
\frac{\delta}{R} \sim \frac{1}{R e} \sqrt{a R e+b\left(\frac{R a}{P r}\right)^{1 / 2}}
$$

or 


$$
\frac{\delta}{R} \sim \frac{1}{R e^{1 / 2}} \sqrt{a+b R i^{1 / 2}}
$$

where $a=e^{-\theta R i}$ and $b=1-e^{-\theta R i}$, with $\theta$ being a parameter, ensure $U \sim(\Omega R)$ for small values of $R i$ (i.e. for forced convection) whereas one obtains $U \sim \sqrt{g \beta \Delta T R}$ for large values of $R i$ (i.e. for natural convection). According to Ri, Eq. (16) provides different scaling estimates. For example, for fully forced convection (i.e. $R i=0)$ Eq. (16) yields,

$$
\frac{\delta}{R} \sim \frac{1}{R e^{1 / 2}}
$$

For $R i \gg 1$ (when natural convection dominates the flow) one obtains:

$$
\frac{\delta}{R} \sim \frac{1}{R e}\left(\frac{R a}{P r}\right)^{1 / 4}
$$

Equation (17b) shows that the effects of rotation sustain even for $R i \gg 1$. Substituting Eq. (16) in Eq. (12b) leads to the following scaling estimate for the mean Nusselt number:

$$
\overline{N u} \sim \frac{R e}{\sqrt{a R e+b\left(\frac{R a}{P r}\right)^{1 / 2}}} f_{1}(R e, R i, P r)
$$

Equation (18) can also be rewritten based on Richardson number using $\operatorname{Ra} / \operatorname{Pr}=\operatorname{Ri} \operatorname{Re}^{2}$ :

$$
\overline{N u} \sim \frac{R e^{1 / 2}}{\sqrt{a+b R i^{1 / 2}}} f_{1}(R e, R i, P r)
$$

Equation (18) offers important physical insights into the influences of $R e, R i$, and $\operatorname{Pr}$ on the mean Nusselt number $\overline{N u}$. In the following section, this scaling predictions will be used for discussing $R e, R i$ and $P r$ effects on the mean Nusselt number.

\section{RESULTS \& DISCUSSIONS}

For each boundary condition configurations, some preliminary simulations have been carried out for different $R e$ values at $R i=0.1$. It has been observed that both rotating top and bottom cover configurations yield the same numerical value of the mean Nusselt number $(\overline{N u})$ when the thermal boundary conditions are kept unaltered, as shown in Fig. 4. This indicates that heat transfer rate for a given set of thermal boundary conditions remains insensitive to the orientation of the rotating end wall. For this reason, only $\mathrm{C} 1$ and $\mathrm{C} 2$ configurations will henceforth be considered in this analysis. 


\subsection{Reynolds Number effects}

The variation of the mean Nusselt number $\overline{N u}$ with Reynolds number $R e$ for $R i=0$ (i.e. purely forced convection) and 0.1 (i.e. a representative mixed convection case) at $\operatorname{Pr}=100$ is shown in Fig. 5. It can be seen from Fig. 5 that $\overline{N u}$ increases with increasing $R e$ for both $\mathrm{C} 1$ and $\mathrm{C} 2$ configurations. This is consistent with the scaling estimate of the mean Nusselt number given by Eq. (18) which also suggests that $\overline{N u}$ is expected to increase with increasing Re. In addition, it can be observed from Fig. 5 that the values of $\overline{N u}$ in the $\mathrm{C} 1$ configuration for $R i=0.1$ (i.e. a representative mixed convection case) are smaller than those obtained in the case of $\mathrm{C} 2$ configuration with the same nominal values of Reynolds number, $R e$ while both $\mathrm{C} 1$ and $\mathrm{C} 2$ configurations exhibit the same numerical value of $\overline{N u}$ for $R i=0$ (i.e. purely forced convection). The orientation of the rotating end wall (whether it is top or bottom) does not affect forced convection in cylindrical enclosures and thus the mean Nusselt number values remain identical for both $\mathrm{C} 1$ and $\mathrm{C} 2$ configurations.

The distributions of non-dimensional swirl velocity component $V_{\phi}(=v H / \alpha)$ along the vertical midplane $(r / R=0.5)$ for both $\mathrm{C} 1$ and $\mathrm{C} 2$ configurations are shown in Fig. 6 for different values $R e$ at $R i=$ 0 (i.e. purely forced convection) and 0.1 (i.e. a representative mixed convection case) at $\operatorname{Pr}=100$. It can be seen from Fig. 6 that the magnitude of $V_{\phi}$ increases with increasing $R e$ for both $\mathrm{C} 1$ and $\mathrm{C} 2$ configurations. In addition, the magnitudes of $V_{\phi}$ for the $\mathrm{C} 2$ configuration are greater than those obtained in the case of $\mathrm{C} 1$ configuration with the same nominal values of $R e$ for $R i=0.1$. This suggests that the advective transport is stronger in the $\mathrm{C} 2$ configuration than in the $\mathrm{C} 1$ configuration, which is reflected in the higher values of the mean Nusselt number $\overline{N u}$ in the $\mathrm{C} 2$ configuration (see Fig. 5).

Figures 5 and 6 suggest a strengthening of advective transport with increasing Reynolds number, which leads to an increase in heat transfer rate within the enclosure. This can also be explained by integrating convective heat transport through the boundary layer thickness on the horizontal walls: 


$$
Q_{c o n v}=Q_{a d v}+Q_{d i f f}=\int_{0}^{\delta} \rho c_{p} u \Delta T d z-\int_{0}^{\delta} k(\partial T / \partial r) d z
$$

where

$$
\begin{aligned}
& Q_{a d v}=\int_{0}^{\delta} \rho c_{p} u \Delta T d z \sim \rho c_{p} U \Delta T \delta \\
& Q_{\text {diff }}=-\int_{0}^{\delta} k\left(\frac{\partial T}{\partial r}\right) d z \sim(k \Delta T) \frac{\delta}{R}
\end{aligned}
$$

where $\delta$ is the hydro-dynamic boundary layer thickneess on the horizontal walls. Substituting $U \sim a(\Omega R)+b(\sqrt{g \beta \Delta T R})$ and Eq. (16a) into Eqs. (21a) and (21b) yields the following scaling estimates for the magnitudes of $Q_{a d v}$ and $Q_{d i f f}$ :

$$
\begin{aligned}
& Q_{a d v} \sim(k \Delta T) R e^{0.5} \operatorname{Pr}\left[a+b R i^{1 / 2}\right]^{3 / 2} \\
& Q_{d i f f} \sim(k \Delta T) \frac{1}{\sqrt{R e}}\left[a+b R i^{1 / 2}\right]^{1 / 2}
\end{aligned}
$$

Eqs. (22a) and (22b) indicate that the advective (diffusive) heat transport $Q_{a d v}$ strengthens (weakens) with increasing $R e$ as observed in Figs. 5 and 6. For this reason, the mean Nusselt number $\overline{N u}$ increaes with increasing $R e$ for both $\mathrm{C} 1$ and $\mathrm{C} 2$ configuratiuons (Fig. 5).

\subsection{Richardson Number effects}

The variations of the mean Nusselt number $\overline{N u}$ with Richardson number $R i$ are presented in Fig. 7 for both $\mathrm{C} 1$ and $\mathrm{C} 2$ configurations for different $R e$ values at $\operatorname{Pr}=100$. Figure 7 shows that the variations of $\overline{N u}$ with $R i$ for $\mathrm{C} 1$ and $\mathrm{C} 2$ configurations are qualitatively different from each other. For $\mathrm{C} 1$ configuration, where the rotating top cover is hotter than the bottom one, $\overline{N u}$ monotonically decreases with an increase in $R i$ according to the scaling estimation given by Eq. (19). However, as observed from Fig.7, the mean Nusselt number $\overline{N u}$ increases mildly with an increase in $R i$ before becoming mostly insensitive to the changes in Richardson number $R i$ in the $\mathrm{C} 2$ configuration, where the rotating top cover is colder than the bottom one. The difference in behaviours between $\mathrm{C} 1$ and $\mathrm{C} 2$ configurations can be explained in the following manner. For mixed convection (i.e. $R i>0$ ), the relative strengths of inertial, 
buoyancy and viscous forces determine the flow and heat transfer behaviours. For $R i=0$, which corresponds to purely forced convection, the flow is governed by the inertial and viscous forces. The influence of buoyancy force starts to strengthen with increasing $R i$, and therefore the competition between buoyancy and viscous forces becomes increasingly important with increasing Ri. However, the $\mathrm{C} 1$ configuration, where the top cover is hotter than the bottom one, represents a stable condition where the lighter hot fluid sits on top of heavier cold fluid so the effects of natural convection remain significant only close to the heated top cover. In this configuration, the flow induced by top wall rotation is opposed by stable stratification of fluid, and thus the net advective transport weakens and the mean Nusselt number decreases with increasing $R i$. On the other hand, in the case of $\mathrm{C} 2$ configuration, where the rotating top cover is colder than the bottom one, represents an inherently unstable configuration where the heavier cold fluid sits on top of lighter hot fluid. Thus, buoyancy force plays a more important role in the $\mathrm{C} 2$ configuration than that in the case of $\mathrm{C} 1$ configuration, and this buoyancy-induced flow in the $\mathrm{C} 2$ configuration aids the fluid motion initiated by the rotating end cover for small values of $R i$, which leads to a marginal increase in the mean Nusselt number $\overline{N u}$. However, the velocity arising from natural convection can be scaled as $\sqrt{g \beta \Delta T H}$ whereas the magnitude vertical velocity induced by the rotation of top wall can be taken to scale with $\Omega R$ and the ratio of these two velocity scales yields $\sqrt{g \beta \Delta T H} / \Omega R \sim R i^{-0.5}$ for an aspect ratio equal to unity. This suggests that the contribution of natural convection is expected to be weak in comparison to the forced convection component for the range of $R i$ considered here. As a result, a change in $R i$ has a marginal impact on the thermal boundary layer transport beyond a mild variation in $\overline{N u}$ due to the introduction of natural convection in comparison to a pure-conduction condition (i.e. $R i=0$ ). As a result, the mean Nusselt number $\overline{N u}$ remains almost insensitive to the variation of $R i$ in the $\mathrm{C} 2$ configuration especially for high $R i$ values, as observed from Fig. 7.

The observations made from Fig. 7 can further be explained from the distributions of the nondimensional temperature $\theta$ and swirl velocity component $V_{\phi}$ along the vertical mid-plane, which are presented in Fig. 8 for different $R i$ values for both $\mathrm{C} 1$ and $\mathrm{C} 2$ configurations at $R e=1000$ and $P r=$ 
100. It can be observed from Fig. 8a that the thermal boundary layer thickness on the bottom cover increases with increasing Richardson number in the case of $\mathrm{C} 1$ configuration. This is also consistent with the scaling estimate of $\delta_{t h}$ given by Eq. (16b). This thickening of boundary layer with increasing $R i$ leads to a decrease in the Nusselt number $\left(\overline{N u} \sim h R / k \sim R / \delta_{t h}\right)$ as shown in Fig. 7. A decrease in Nusselt number with increasing Richardson number bears the signature of the wakening of advective transport. This can further be substantiated from the distribution of the non-dimensional swirl velocity component along the vertical mid-plane (i.e. $r / R=0.5$ ) in Fig. $8 \mathrm{a}$., which shows that the magnitude of $V_{\phi}$ increases with increasing $R i$ only in the vicinity of the hot (top) cover in the case of $\mathrm{C} 1$ configuration. However, the magnitude of $V_{\phi}$ decreases with increasing $R i$ towards the bottom of the container. As a result of this, advective transport weakens and the mean Nusselt number $\overline{N u}$ decreases with increasing $R i$ in the $\mathrm{C} 1$ configuration. By contrast, it can be seen from Fig. $8 \mathrm{~b}$ that the thermal boundary layer thickness on the bottom cover is not significantly influenced by the variation of $R i$ in the $\mathrm{C} 2$ configuration. It can further be seen from the distribution of the non-dimensional swirl velocity component along to vertical mid-plane (i.e. $r / R=0.5$ ) that the magnitude of $V_{\phi}$ does not get significantly affected by a variation of $R i$, which suggests that a change in $R i$ has a marginal influence on the strength of advective transport within the enclosure in the $\mathrm{C} 2$ configuration. As a result of this, the mean Nusselt number $\overline{N u}$ remains mostly insensitive to the changes in Richardson number $R i$ in the case of C2 configuration (see Fig. 7).

\subsection{Prandtl Number effects}

The variations of the mean Nusselt number $\overline{N u}$ with $\operatorname{Pr}$ for both $\mathrm{C} 1$ and $\mathrm{C} 2$ configurations are shown in Fig. 9 for $R e=1000$ and 3000 at $R i=0.1$ (i.e. a representative mixed convection case). Figure 9 indicates that Prandtl number $P r$ has an important influence on $\overline{N u}$ in both $\mathrm{C} 1$ and $\mathrm{C} 2$ configurations, and the mean Nusselt number $\overline{N u}$ increases with increasing $P r$. This can be expected from the scaling estimation given by Eq. (19) because the function $f_{1}$ is expected to increase with increasing $\operatorname{Pr}$. The strengthening of advective transport with increasing $\operatorname{Pr}$ can be explained from the distributions of $\theta$ and $V_{\phi}$ along the vertical mid-plane, which are shown in Fig. 10 for $R e=1000$ at $R i=0.1$. It is apparent 
from Fig. 10 that the thermal boundary layer thickness decreases with increasing $\operatorname{Pr}$ for both $\mathrm{C} 1$ and $\mathrm{C} 2$ configurations, which in turn acts to increase the mean Nusselt number $\overline{N u} \sim R / \delta_{t h}$. Furthermore, the magnitude of non-dimensional swirling velocity component $V_{\phi}$ rises with increasing $\operatorname{Pr}$ for both configurations. This indicates that the advective transport strengthens with increasing $P r$, which is consistent with the findings based on Fig. 9. The strengthening of advective transport with increasing $\operatorname{Pr}$ can also be explained by the scaling estimate of $Q_{a d v}=\int_{0}^{\delta} \rho c_{p} u \Delta T d z$ given by Eq. (22a). Equation (22a) explicitly shows that advective transport strengthens with increasing $\operatorname{Pr}$ and accordingly, the mean Nusselt number increases with increasing $\operatorname{Pr}$ for both $\mathrm{C} 1$ and $\mathrm{C} 2$ configurations, as observed in Fig. 9 .

\subsection{Flow regimes}

The variation of non-dimensional stream functions $\Psi=\psi / \alpha$ for both $\mathrm{C} 1$ and $\mathrm{C} 2$ configurations are shown in Fig. 11 for different $R i$ and $P r$ values at $R e=1000$. It is apparent from Fig. 11 that the flow pattern in the $\mathrm{C} 1$ configuration is significantly different from those in the $\mathrm{C} 2$ configuration. This is also in accordance to the findings based on Figs. 7 and 8, which demonstrate that $\mathrm{C} 1$ and $\mathrm{C} 2$ configurations exhibit different behaviours in response to the changes in Richardson number. For $R i=0$ (i.e. purely forced convection), both $\mathrm{C} 1$ and $\mathrm{C} 2$ configurations exhibit same flow structures, which is consistent with the results shown in Figs. 5-10. It can be observed from Fig. 11 that a one-cell flow structure is observed in the case of $\mathrm{Cl}$ configuration for the range of $R i$ and $\operatorname{Pr}$ considered here, whereas the flow pattern changes depending on $R i$, and $P r$ in the $\mathrm{C} 2$ configuration at $R e=1000$. The same behaviour has also been observed for different Reynolds number values. Based these observations, a flow regime diagram has been proposed here for the $\mathrm{C} 2$ configuration for the range of $R i, \operatorname{Pr}$ and $R e$ analysed in this study. According to this diagram, the flow patterns in the $\mathrm{C} 2$ configuration are classified into three different zones in terms of Richardson number $R i$ and Peclet number $P e=R e P r$ (which characterises the ratio of advective thermal transport to thermal diffusive transport) as shown in Fig. 12. The region, where Richardson number is small (i.e. $R i<0.1$ ), is termed as the Regime 1 , which exhibits one-cell flow pattern. The Regime 2 is characterised by $0.1 \leq R i<0.7$ and $10^{4}<P e$ where a secondary circulation appears on the bottom wall (i.e. hot wall) corner. The size of the circulation at the corner of 
the bottom wall (i.e. hot wall) increases with increasing $R i$ (i.e. as the natural convection strengthens), whereas it decreases with increasing $P e$ (i.e. as thermal advection starts to dominate over thermal conduction). Finally, the parameter space given by $0.7 \leq R i$ and $P e \leq 10^{4}$ is defined as the Regime 3 . A two-cell flow pattern occurs in the Regime 3. It needs to be highlighted that the boundaries which distinguish one regime from another in Fig. 12 are based on the observations made from simulation results. As such, these boundaries should not be treated rigidly but need to be considered only in an order of magnitude sense.

\subsection{The mean Nusselt number correlation}

Based on the simulation results, the following correlation for the mean Nusselt number $\overline{N u}$ have been proposed by Turan et al. [15] for the $\mathrm{C} 1$ configuration in the parameter range given by $0 \leq R i \leq 1$, $500 \leq \operatorname{Re} \leq 3000$ and $10 \leq \operatorname{Pr} \leq 500:$

$$
\overline{N u}=1+k_{0} R e^{m_{0}}
$$

where $k_{o}$ and $m_{o}$ are the correlation parameters, which are listed in Table 4. The correlation given by Eq. (23) has also been adopted here for the $\mathrm{C} 2$ configuration but the values of $k_{o}$ and $m_{o}$ are different to those in the case of $\mathrm{C} 1$ configuration. The predictions of the correlation given by Eq. (23) are compared to the numerical results in Fig. 13 for both $\mathrm{C} 1$ and $\mathrm{C} 2$ configurations. Figure 13 demonstrates that the correlation, given by Eq. (23), satisfactorily captures both qualitative and quantitative variations of $\overline{N u}$ with for the range of $R i, R e$ and $\operatorname{Pr}$ analysed in this study.

\section{CONCLUSIONS}

The effects of Richardson, Reynolds, and Prandtl numbers on heat and momentum transport in steady state laminar mixed convection in cylindrical enclosures with a rotating end cover and an aspect ratio (height: radius) of unity (i.e. $A R=H / R=1$ ) have been numerically analysed for different boundary conditions for top and bottom walls. It has been found that the heat transfer rate for a given set of thermal boundary conditions remains insensitive to whether the top or bottom cover is rotated. For this reason, only $\mathrm{C} 1$ (where the top rotating wall is heated) and $\mathrm{C} 2$ (where the top rotating wall is cooled) 
configurations have been considered in this analysis. It has been found out that Reynolds number and Prandtl number dependences of the mean Nusselt number remain qualitatively similar for both $\mathrm{C} 1$ and $\mathrm{C} 2$ configurations, whereas the variation of $\overline{N u}$ with $R i$ is qualitatively different in these configurations. The mean Nusselt number $\bar{N} \bar{u}$ increases with increasing $\operatorname{Re}$ and $\operatorname{Pr}$ for both $\mathrm{C} 1$ and $\mathrm{C} 2$ configurations. However, $\overline{N u}$ demonstrates a monotonically decreasing trend with increasing $R i$ in the $\mathrm{C} 1$ configuration, whereas $\overline{N u}$ exhibits a mild increase with increasing $R i$ before becoming mostly insensitive to the changes in Richardson number $R i$ in the $\mathrm{C} 2$ configuration. It has been also observed that the $\mathrm{C} 1$ configuration exhibits one-cell flow structure for the range of $R i, \operatorname{Pr}$ and $R e$ considered here, whereas the flow pattern in the $\mathrm{C} 2$ configuration changes significantly depending on $R i, \operatorname{Pr}$ and $R e$ values. A flow regime diagram has been proposed for the $\mathrm{C} 2$ configuration for the range of $R i, \operatorname{Pr}$ and $R e$ analysed in this study. According to this diagram, the flow patterns are classified into three different zones; Regime 1: one-cell flow pattern, Regime 2: A secondary circulation appears on the bottom wall (i.e. hot wall) corner and Zone 3: Two-cell flow pattern. Finally, based on the simulation results, a correlation for the mean Nusselt number has been proposed for both $\mathrm{C} 1$ and $\mathrm{C} 2$ configurations, and this correlation has been shown to satisfactorily capture both qualitative and quantitative variations of $\overline{N u}$ with for the range of $R i, R e$ and $\operatorname{Pr}$ considered in this analysis.

\section{ACKNOWLEDGEMENTS}

This study was supported by Newton Research Collaboration Programme and is hereby gratefully acknowledged. 


\section{REFERENCES}

[1] P. J. Zandbergen and D. Dikstra, Von Karman swirling flows, Ann. Rev. Fluid Mech. 19 (1987), 465-491.

[2] H. U. Vogel, Experimentelle Ergebnisse über die laminare Strömung in einem zylindrischen Gahause mit darin rotieren-der Scheibe. MPI Bericht 6, 1968.

[3] H. U. Vogel, Rückströmungsblasen in Drallsströmungen. Festschrift 50 Jahre Max-Planck-Institut für Strömungsforschung 1925-1975, 1975.

[4] B. Ronnenberg, Ein selbstjustierendes 3-Komponenten-Laserdoppleranemometer nach dem Vergleichsstrahlverfahren, angewandt für Untersuchungen in einer stationaren sylinder-symmetrischen Drehströmung mit einem Rückstromgebiet. MPI Bericht 20, 1977.

[5] M. Bertela, F. Gori, Laminer flow in a cylindrical container with a rotating cover, J. Fluids Eng. 104 (1982) 31-39.

[6] M.P. Escudier, Observations of the flow produced in a cylindrical container by rotating endwall, Experiments in Fluids 2 (1984) 189-196.

[7] K. Fujimura, H.S. Koyama, and J.M. Hyun, An experimental study on vortex breakdown in a differentially-rotating cylindrical container, Exp. Fluids 36 (2004) 399-407.

[8] H.J. Lugt, H.J. Haussling, Axisymmetric vortex breakdown in rotating fluid within a container, Trans. ASME J. Appl. Mech. 49 (1982) 921-923.

[9] J.M. Lopez, Axisymmetric vortex breakdown: Part1. Confined swirling flow, J. Fluid Mech. 221(1990) 533-552.

[10] W. N. Kim and J. M. Hyun, Convective heat transfer in a cylinder with a rotating lid under stable stratification, Int. J. Heat and Fluid Flow 18 (1997) 384-388.

[11] C.H. Lee, J.M. Hyun, Flow of a stratified fluid in a cylinder with a rotating lid, Int. J. Heat and Fluid Flow 20 (1999) 26-33.

[12] R. Iwatsu, Flow pattern and heat transfer of swirling flows in cylindrical container with rotating top and stable temperature gradient, Int. J. Heat and Mass Transfer 47 (2004) 2755-2767.

[13] J.C.F. Pereira and J.M.M. Sousa, Confined vortex breakdown generated by a rotating cone, J. Fluid Mechanics 385 (1999) 287-323. 
[14] M. P. Escuider, J. O'Leary, R.J. Poole, Flow produced in a conical container by a rotating end wall, Int. J. of Heat and Fluid Flow 28 (2007) 1418-1428.

[15] O. Turan, S. Yigit and N. Chakraborty, Numerical investigation of mixed convection of Bingham fluids in cylindrical enclosures with heated rotating top wall, Int. J. Heat and Mass Transfer 108 (2017) $1850-1869$.

[16] S. V. Patankar, Numerical Heat Transfer and Fluid Flow, Hemisphere, Washington, D.C, 1980.

[17] P. Yu, T.S. Lee, Y. Zeng and H.T. Low, Characterization of flow behavior in an enclosed cylinder with a partially rotating end wall, Physics of Fluids 19 (2007) 057104.

[18] R. Bessaih, A. Boukhari and P. Marty, Magnetohydrodynamics stability of a rotating flow with heat transfer, Int. Communication in Heat and Mass Transfer 36 (2009) 893-901.

[19] A. Guha, S. Sengupta, Analysis of von Kármán's swirling flow on a rotating disc in Bingham fluids, Phys. Fluiids 28 (2016) 013601.

[20] O. Turan, N. Chakraborty, R. J. Poole, Laminar natural convection of Bingham fluids in a square enclosure with differentially heated side walls, J. Non-Newtonian Fluid Mech. 165(2010) 901-913. 
Table 1. Summary of the existing analyses on the flow induced by a rotating one of the covers of a cylindrical container involving Newtonian fluids.

\begin{tabular}{|c|c|c|c|c|c|c|c|}
\hline Authors & Year & Type & Rotating Wall & $\begin{array}{l}\text { Thermal Boundary } \\
\text { Conditions }\end{array}$ & $\begin{array}{c}\text { Investigation } \\
\text { Area }\end{array}$ & $A R=H / R$ & $R i, \operatorname{Re}, P r$ \\
\hline Vogel [2] & 1968 & $\mathrm{E}^{*}$ & Bottom wall & - & Flow characteristics & $1.39 \leq A R \leq 2.12$ & $1000 \leq R e \leq 2870$ \\
\hline Vogel [3] & 1975 & $\mathrm{E}$ & Bottom wall & - & Flow characteristics & - & - \\
\hline Ronnenberg [4] & 1977 & $\mathrm{E}$ & Bottom wall & - & Flow characteristics & - & - \\
\hline Bertela and Gori [5] & 1982 & $\mathrm{~N}^{* *}$ & Top wall & - & Flow characteristics & $0.5 \leq A R \leq 2$ & $R e=100,1000$ \\
\hline Escudier [6] & 1984 & $\mathrm{E}$ & Bottom wall & - & Flow characteristics & $1.5 \leq A R \leq 3.5$ & $1000 \leq R e \leq 3000$ \\
\hline Lugt and Haussling [8] & 1982 & $\mathrm{~N}$ & Top wall & - & Flow characteristics & $A R=1.58$ & $R e=1130,1250,1355$ \\
\hline Lopez [9] & 1990 & $\mathrm{~N}$ & Bottom wall & - & Flow characteristics & $A R=2.5,3.25,3.5$ & $1000 \leq R e \leq 3061$ \\
\hline Kim and Hyun [10] & 1997 & $\mathrm{~N}$ & Top wall & $T_{\text {top }}>T_{\text {bottom }}$ & $\begin{array}{l}\text { Heat transfer } \\
\text { characteristics }\end{array}$ & $A R=2$ & $\begin{array}{c}0.01 \leq R i \leq 10 \\
100 \leq R e \leq 2500 \\
\operatorname{Pr}=0.7\end{array}$ \\
\hline Lee and Hyun [11] & 1999 & $\mathrm{~N}$ & Top wall & $\begin{array}{l}T_{\text {top }}>T_{\text {bottom }} \\
T_{\text {top }}<T_{\text {bottom }}\end{array}$ & $\begin{array}{l}\text { Heat transfer } \\
\text { characteristics }\end{array}$ & $A R=2$ & $\begin{array}{c}-0.01 \leq R i \leq 1^{* * *} \\
\operatorname{Re}=1600 \\
0.1 \leq \operatorname{Pr} \leq 10\end{array}$ \\
\hline Iwatsu [12] & 2004 & $\mathrm{~N}$ & Top wall & $T_{t o p}>T_{b o t t o m}$ & $\begin{array}{l}\text { Heat transfer and flow } \\
\text { characteristics }\end{array}$ & $A R=1$ & $\begin{array}{c}0 \leq R i \leq 1 \\
100 \leq R e \leq 3000 \\
\operatorname{Pr}=1\end{array}$ \\
\hline Escuider et al. [14] & 2007 & $\mathrm{~N}$ & Bottom wall & - & Flow characteristics & $A R=2$ & $\operatorname{Re}=1854,2354,3354$ \\
\hline
\end{tabular}

*E: experimental; **N: numerical; *** Negative (positive) values of $R i$ refer $T_{\text {top }}<T_{\text {bottom }}\left(T_{\text {top }}>T_{\text {bottom }}\right)$ [11]. 
Table 2. The details of the meshes and the numerical uncertainty for the mean Nusselt number $\overline{N u}$ for $R i=0.1$ and $R e=1000$ at $P r=100$.

\begin{tabular}{|c|c|c|c|c|c|c|}
\hline & \multicolumn{3}{|c|}{$\mathrm{C1}$} & \multicolumn{3}{|c|}{$\mathrm{C} 2$} \\
\hline Mesh Details & $\begin{array}{c}\text { M1 } \\
(50 \times 50)\end{array}$ & $\begin{array}{c}\text { M2* } \\
(100 \times 100)\end{array}$ & $\begin{array}{c}\text { M3 } \\
(200 \times 200)\end{array}$ & $\begin{array}{c}\text { M1 } \\
(50 \times 50)\end{array}$ & $\begin{array}{c}\text { M2* } \\
(100 \times 100)\end{array}$ & $\begin{array}{c}\text { M3 } \\
(200 \times 200)\end{array}$ \\
\hline$\Delta_{\text {min,cell }} / R$ & $4.60 \times 10^{-3}$ & $2.30 \times 10^{-3}$ & $1.15 \times 10^{-3}$ & $3.10 \times 10^{-3}$ & $1.55 \times 10^{-3}$ & $0.78 \times 10^{-3}$ \\
\hline$r_{e}$ & 1.0287 & 1.0141 & 1.0070 & 1.0630 & 1.0307 & 1.0152 \\
\hline \multicolumn{7}{|c|}{ Numerical Uncertainty } \\
\hline$\overline{N u}$ & 26.5178 & 25.7924 & 25.5440 & 32.8294 & 32.2667 & 32.0751 \\
\hline$e_{a}(\%)$ & \multicolumn{2}{|c|}{2.7355} & 0.9631 & 1.71 & \multicolumn{2}{|c|}{0.5938} \\
\hline
\end{tabular}

* The mesh which is used for the numerical simulations. 
Table 3. Scaling estimates for characteristic velocity scales.

\begin{tabular}{cccc}
\hline \hline Velocity Scale & Forced Convection & Natural Convection & Mixed Convection \\
\hline$V \sim$ & $\Omega R^{*}$ & $\Omega R^{*}$ & $\Omega R^{*}$ \\
$U \sim$ & $\Omega R^{*}$ & ${\sqrt{g \beta \Delta T R^{* *}}}^{*}$ & $a(\Omega R)+b(\sqrt{g \beta \Delta T R})^{* * *}$ \\
\hline \hline
\end{tabular}

${ }^{*}[19],{ }^{* *}[20],{ }^{* * *} a=e^{-\theta R i}$ and $b=1-e^{-\theta R i}$. 
Table 4. Summary of the mean Nusselt number $\overline{N u}$ correlation functions.

\begin{tabular}{|c|c|}
\hline & $\overline{N u}=1+k_{0} R e^{m_{0}}$ \\
\hline C1 & $10 \leq \operatorname{Pr}<100$ and $0 \leq R i \leq 1$ \\
\hline \multicolumn{2}{|r|}{$k_{0}=(0.015-0.003 \ln P r)+(0.113 \ln P r-0.164) \exp (-\operatorname{Ri}(2.539+1.214 \ln P r))$} \\
\hline \multicolumn{2}{|r|}{$m_{0}=(0.746-0.022 \ln P r)+(0.367 \ln P r-0.83) R i^{(0.229 \ln P r-0.046)}$} \\
\hline \multicolumn{2}{|r|}{$100 \leq P r \leq 500$ and $0 \leq R i \leq 0.5$} \\
\hline \multicolumn{2}{|r|}{$k_{0}=(0.283 \ln P r-0.947) \exp (-\operatorname{Ri}(16.76-1.874 \ln P r))$} \\
\hline \multicolumn{2}{|r|}{$m_{0}=(0.742-0.02 \ln P r)+(1.65-0.172 \ln P r) R i^{(0.9+0.023 \ln P r)}$} \\
\hline \multicolumn{2}{|r|}{$100 \leq \operatorname{Pr} \leq 500$ and $0.5<R i \leq 1$} \\
\hline \multicolumn{2}{|r|}{$k_{0}=(0.065 \ln P r+0.012) \exp (-\operatorname{Ri}(4.187+0.161 \ln P r))$} \\
\hline \multicolumn{2}{|r|}{$\left.m_{0}=(-0.188+0.135 \ln P r)+(0.793-0.025 \ln P r) R i^{(0.107+0.137 \ln P r}\right)$} \\
\hline $\mathbf{C 2}$ & $10 \leq \operatorname{Pr}<100$ \\
\hline \multicolumn{2}{|r|}{$k_{0}=(0.056+0.012 \ln P r)+(0.129 \ln P r-0.225) \exp (-\operatorname{Ri}(3.368+3.123 \ln P r))$} \\
\hline \multicolumn{2}{|r|}{$m_{0}=(0.757-0.050 n P r)+(0.422 \ln P r-0.897) R i^{(0.118 \ln P r-0.083)}$} \\
\hline \multicolumn{2}{|r|}{$100 \leq \operatorname{Pr} \leq 500$} \\
\hline \multicolumn{2}{|r|}{$k_{0}=(0.207 \ln P r-0.605) \exp (-R i(0.177+0.004 \ln P r))$} \\
\hline$m_{0}$ & $(0.683-0.008 \ln P r)+(0.006+0.004 n P r) R i^{(0.590+0.016 \ln P r)}$ \\
\hline
\end{tabular}




\section{FIGURE CAPTIONS}

Fig. 1. Schematic diagrams of the simulation domain and different boundary condition cases where $T_{H}$ and $T_{C}$ are the hot and cold wall temperatures.

Fig. 2. Comparison of present simulation results with benchmark results by Iwatsu [12].

Fig. 3. Comparison of computed streamlines and experimental flow visualisation [6] for $R e=1854$, $A R=H / R=2$.

Fig. 4. Variation of mean Nusselt number $\overline{N u}$ with Reynolds number $R e$ for different boundary conditions at $R i=0.1$ and $\operatorname{Pr}=100$.

Fig. 5. Variation of mean Nusselt number $\overline{N u}$ with Reynolds number $R e$ for $R i=0$ and 0.1 at $\operatorname{Pr}=100$.

Fig. 6. Variation of non-dimensional swirl velocity component $V_{\phi}$ along the vertical mid-plane (i.e. $r / R$ $=0.5$ ) for different Reynolds number $R e$ values for $R i=0$ and 0.1 at $\operatorname{Pr}=100$.

Fig. 7. Variation of mean Nusselt number $\overline{N u}$ with Richardson number $R i$ for both $\mathrm{C} 1$ (red) and $\mathrm{C} 2$ (blue) configurations for different $R e$ values at $\operatorname{Pr}=100$.

Fig. 8. Variations of non-dimensional temperature $\theta$ and swirl velocity $V_{\phi}$ along the vertical mid-plane (i.e. $r / R=0.5$ ) for both $\mathrm{C} 1$ and $\mathrm{C} 2$ configurations at $R e=1000$ and $\operatorname{Pr}=100$.

Fig. 9. Variation of mean Nusselt number $\overline{N u}$ with Prandtl number $P r$ for both $\mathrm{C} 1$ and $\mathrm{C} 2$ configurations for $R e=1000$ and 3000 at $R i=0.1$.

Fig. 10. Variations of non-dimensional temperature $\theta$ and swirl velocity $V_{\phi}$ along the vertical mid-plane (i.e. $r / R=0.5$ ) for both $\mathrm{C} 1$ and $\mathrm{C} 2$ configurations at $R e=1000$ and $R i=0.1$.

Fig. 11. Distributions of non-dimensional stream function $\Psi=\psi / \alpha$ for both $\mathrm{C} 1$ and $\mathrm{C} 2$ configurations for different $R i$ and $\operatorname{Pr}$ values at $R e=1000$.

Fig. 12. Flow regime diagram on $R i-\operatorname{Pr} \times \operatorname{Re}$ plane in the case of $\mathrm{C} 2$ configuration.

Fig. 13. Comparison between $\overline{N u}$ obtained from the simulations with the predictions of Eq. (23) for both $\mathrm{C} 1$ and $\mathrm{C} 2$ configurations. 